\title{
Spin Quantum Optic Ion Off Let Sugato- Chandrasekhar Spin Atom Excitation
}

\author{
Sugato Ghosh \\ Department of Physics, JLD College of Engineering \& Management, Under Maulana Abul Kalam Azad University of Technology (formally \\ West Bengal University of Technology), West Bengal, India
}

\begin{abstract}
I performed theoretically spin quantum optic ion off let to classic to non classic territory onto spin Sugato- Chandrasekhar atom in build signature of off bound blue light transition onto it extension off let expansion with fermion ion optic into zero space Sugato Limit.
\end{abstract}

Keywords: Sugato-Chandrasekhar spin atom, Sugato Limit

\section{Introduction}

The Limit off let boundary generating measuring with onto is signature of neighbour state is off let function in ground generation gauge entire theory of state trace off break off set ion generation. It has had to be the dense fermions ion optic within build atom signature with $\lambda$ the dimensional space Hilbert Chandrasekhar limit with mass of generating gaseous optic ion into the condensed boson degenerating off let phase regenerating let break generation with to be a gauge light optic colour transition to the in build signature of $\Pi$ space $\lambda$ gyrative expansion with onto be a High break symmetry with gauge zero off let off break symmetry.

The central fact concerning normal stars (quantum optic ion) is the role which radiation pressure plays as a factor in their hydrostatic equilibrium. Precisely the equation governing the hydrostatic equilibrium of a star

$\frac{\mathrm{dp}}{\mathrm{dr}}=-\frac{\mathrm{GM}(\mathrm{r})}{\mathrm{r}^{2}} \rho$

Where $\rho$ denotes the total pressure, $\mathrm{p}$ the density and $\mathrm{M}(\mathrm{r})$ is the mass interior to a sphere of radius $\mathrm{r}$. There are two contributions to the total pressure $\mathrm{P}$, that due to the material and that due to the radiations. On the assumption that the matter is in the state of a perfect gas in the classical Maxwell a sense, the material on the gas pressure is given by

$\mathrm{p}_{\text {gas }}=\frac{\mathrm{K}}{\mu \mathrm{H}} \rho \mathrm{T}$

Where $\mathrm{T}$ is the absolute temperature $\mathrm{K}$ is the Boltzmann constant and $\mu$ is the mean molecular weight (which under normal stellar conditions is $\sim 1.0$ ) the pressure due to radiation is given by

$\mathrm{p}_{\text {red }}=\frac{1}{3} \alpha \mathrm{T}^{4}$

Where $\alpha$ denotes Stefan's radiation constant [1].

It has had the $\beta_{\mathrm{i}}, \beta_{\mathrm{j}}, \beta_{\mathrm{k}}$ of let to be the line generator light off let with to the response of quantum pulse [4] generating space let off the optic ion generation with classic inertia onto be non-classic spin atom off generating quantum decoherence with the space accusation off string
$\mathrm{H}-\mathrm{I}$ let $\mathrm{M}$ brans let generating gauge symmetry spontaneous.

The limit of Chandrasekhar build generator with $T_{i, j, k}$ space $\mathrm{L}^{2}$ Hilbert with the wave degenerating onto the rotating universe.

The complex spin fermion with brans homogeneous with let space accusation to the limit of colour optic scatter the ion generation with two senses of the light comes out with the debris of spin with dense mode trace $\gamma_{\mathrm{p}_{1}} \ldots \ldots \ldots \ldots \ldots \ldots \gamma_{\mathrm{p}_{\mathrm{n}}}$ multi dimensional space generation and the let off let generating space region to the dynamic spin with analog of expansion.

\section{Chandrasekhar Limit Molasses with gravitation}

The limit off let Chandrasekhar with onto the gyrative synchronized ion build generator with the light spin optic ion into the colour band transition with the function optic annihilation of elastic stretch with well defined the domain of limit colour $\mathrm{Z}_{\lambda_{\text {red }}} \leq \beta \leq \mathrm{Z}_{\lambda_{\text {blue }}}$ into the transition with the optic shadow of image stress with sequence of annihilation.

The defined functional annihilation $\beta_{\mathrm{i}, \mathrm{j}, \mathrm{k}}(\varphi)$ is the integer of chosen function of quantum optic spin $\varphi$ with the interchangeability pick band tolerance with maximum optic stretch probability to minimum stretch probability. The function $\varphi \rightarrow$ monotonic $\mathrm{x}$ inverse of limit with gauge turbulent to space to limit of off bond expansion with system boundary constant perturbation limit C of SugatoChandrasekhar spin atom to its existence.

The $\Lambda$ is the dot guest annihilation of rotating spin with the limit of instant to limit of space infinity off let rotating universe.

The function domain $\mathrm{p}_{1} \ldots \ldots \ldots . \mathrm{p}_{\mathrm{n}}$ of " Sugato Soft weak gravitational wave generation" with the colour of positive $\lambda_{i, j, k}(\varphi)$ function of quantum optic ion of function onto the molasses of "Sugato Soft Weak gravitational wave generation with the colour transformation in build building generation of each polar strength of space Hilbert with the lifted let on accreditation with gyrative the function integral with limit 


\section{International Journal of Science and Research (IJSR)}

ISSN (Online): 2319-7064

Index Copernicus Value (2013): 6.14 | Impact Factor (2015): 6.391

$\frac{1}{\beta_{1}} \mathrm{f}(\mathrm{x}+\Delta \mathrm{h})-\mathrm{f}(\mathrm{x}) \ldots \ldots \ldots \ldots \ldots \frac{1}{\beta_{\mathrm{n}}} \mathrm{f}(\mathrm{x}+\Delta \mathrm{h})-\mathrm{f}(\mathrm{x})$

Where the elastic expansion is to be into the "Sugato Soft weak gravitational wave generation" in phase of quantum optic ion off let spin.

The let of wave function generating kitten to the state gyrative signature of low wave length blue light with high energy excitation off let off bound with maximum limit of "Sugato soft weak gravitation wave generation" with building generator of signature of expansion.

\section{Off let intangible ribbon with annihilation of Hilbert Decoherence}

In the late 1960 s and the 1970 s candidates materials for blue light emitted with to be developed LED based on materials [2]. The growth of large bulk crystals with the alignment with the high quality semi conductivity into the nanostructure off let off set growth generation of "homoepitaxy" or "hetroepitaxy" with break generation of substrate crystal.

Off let high performance GaN p-n junction blue / UV-LED with encouraging current voltage (I-V) Characteristic. This was demonstrated in 1989 and was the first such device in the world [2].The LED exhibited blue light emission with a low current injection $(<20 \mathrm{~mA})$ and additional near UV light emission with a high current injection [3].

The off let boundary with extended the classic to off bound non classic decoherence steam off let generation of ion optic with the bound symmetry with the integral signature of the exponential limit with optic tangential with the off let off set signature of ribbon generator with photonic break generation [3] with generated enlargement molasses of pseudo colour blue with the limit gauge

$\left|g\left(\lambda_{i, j, k}+\Delta 0\right)-g_{\varphi(r, \theta)}(\lambda-\Delta 0)\right|>\frac{1}{H}$

Where $\mathrm{H}$ off let gyrative signature of soft Hilbert with the "Sugato Soft Weak Gravitational wave generation"[7] with fine superposition of expansion femto second $\Delta 0$ with the convergent to the high tensile blue null set to the monotonic divergent $t$ limit with gauge optic

$\left|\left(f_{1} \ldots \ldots \ldots \ldots f_{n}+\Delta 0_{i}+\Delta 0_{j}+\Delta 0_{k}\right)\right| \leq f_{1}\left(a_{1}\right)-f_{1}(b)+f_{2}\left(a_{2}\right)-$

$\mathrm{f}_{2}(\mathrm{~b})+\ldots \ldots . .+\mathrm{f}_{\mathrm{r}}\left(\mathrm{a}_{\mathrm{r}}\right)-\mathrm{f}_{\mathrm{r}}(\mathrm{b})$

Where the choice of integral function with space interval of each $1 / 2$ spins optic rotation of Sugato molasses into the divergent optic ion function $\left(a_{1} \ldots \ldots \ldots a_{r}\right)$ with the preferable optic point of each annihilation with continuous probability $\zeta$ with a continuous generative function.

The upper bound the limit of expansion of ion optic with the reappeared ribbon with to be total variation of pulse relativity [4] with maximum annihilation floating point of each gyrative optic ion into decoherence stage.
Sugato Soft weak gravitational wave generator off let blue tangential zero space Sugato Limit

Gyrative generator of off let blue building $\lambda$ (wave length) onto be low value high energy reorganization with the variable limit of expansion space vacuue to break generation off bound symmetry with let on to colour transition with continuum relativity [4] generative Sugato soft weak gravitation wave generation with off bound signature with annihilation of band generation $\mu$ to coherence with dense mode off let $\left[\rho^{+}\right]^{+}$off generative signature.

Let $\theta$ is to be the there should wave frequency with tangential break rotational symmetry $\left[\theta_{\mathrm{i}, \mathrm{j}, \mathrm{k}}\right]^{+}$annihilation phase shift [5] $\Pi$ to symmetry generator $3 \Pi / 2$ [6] maximum orbital oscillation with onto the signature of space zero with off bound gyrative fermion ion with building signature space zero onto space vacuue off bound spin 1/2 integral Sugato limit of Quantum optic ion.

Spontaneous Charil turbulent inertia classis to non classic quantum fluid flow of wave d state

The spontaneous charil turbulent ingredient oblong optic coherence build signature classic to non classic territory onto the bridge building generator of $d$ state wave stretch being turbulence with onto signature of ion generator coherence with fermion stretch optic multitask annihilation off bound break.

The quantum fluid ion is generator onto the gyrative blue light stretch optic colour transformation onto the zero [4] dimensional matrix with pulse rotation with sense off let sensitivity with ion optic rotation.

\section{Conclusion}

In this paper I work on Chandrasekhar limit with off let spin atom and It had had molasses with gyration. It is also discussed off let intangible ribbon with annihilation of Hilbert decoherence space. Sugato soft weak gravitational wave generation off let to the blue tangential zero space Sugato Limit. It is also spontaneous charil with turbulent inertia classic to non classic quantum fluid flow of wave d state.

\section{Reference}

[1] S.Chandrasekhar Nobel Lecture 1983

[2] H.Matsunami, M.Ikeda, A Suzuki and T.Tanka SiC Blue LED's by Liquid Phase cpitavy, "IEEE Trans. Electro Devices, ED-24, 958(1977)

[3] I. Akasaki Nobel Lectures 2014

[4] S.Ghosh Theory Of Relativity on Pulse Quantum Phenomenon (IJPR) Vol-3 Issue-5, Dec 2013, 21-32

[5] S.Haroche iopPhy ScT137(20090014014)

[6] S.Ghosh Theory on Pulse Phenomenon in the Rydberg atom into the oscillation orbit in the Hilbert atom (IJPR) Vol-5, Issue-6, Dec 2015, 15-29

[7] S.Ghosh Quantum Optic ion with sensational Pulse (IJPR) Vol-6, Issue-2, Apr 2016, 9-14 